\title{
Investigation of the Genomic and Pathogenic Features of the Potentially Zoonotic Streptococcus parasuis
}

\author{
Jianping Wang ${ }^{1,+} \mathbb{D}$, Xueli Yi ${ }^{2,+}$, Pujun Liang ${ }^{1,+}$, Yuanmeihui Tao ${ }^{1}$, Yan Wang ${ }^{1}$, Dong Jin ${ }^{1}$, Bin Luo ${ }^{2}$, Jing Yang ${ }^{1}$ \\ and Han Zheng ${ }^{1, *}$ \\ 1 State Key Laboratory of Infectious Disease Prevention and Control, National Institute for Communicable \\ Disease Control and Prevention, Chinese Center for Disease Control and Prevention, Changping, \\ Beijing 102206, China; wangjianping@icdc.cn (J.W.); liangpujun1997@163.com (P.L.); \\ taoyuanmeihui@163.com (Y.T.); wangyan@icdc.cn (Y.W.); jindong@icdc.cn (D.J.); yangjing@icdc.cn (J.Y.) \\ 2 The Affiliated Hospital of Youjiang Medical University for Nationalities, Clinical College of Youjiang Medical \\ University for Nationalities, Youjiang 533000, China; 1566@ymcn.edu.cn (X.Y.); 1558@ymcn.edu.cn (B.L.) \\ * Correspondence: zhenghan@icdc.cn \\ + Those authors contributed to the manuscript equally.
}

check for updates

Citation: Wang, J.; Yi, X.; Liang, P.; Tao, Y.; Wang, Y.; Jin, D.; Luo, B.; Yang, J.; Zheng, H. Investigation of the Genomic and Pathogenic Features of the Potentially Zoonotic Streptococcus parasuis. Pathogens 2021, 10, 834. https://doi.org/10.3390/ pathogens10070834

Academic Editors: Lawrence S. Young and Angela H. Nobbs

Received: 21 May 2021

Accepted: 30 June 2021

Published: 2 July 2021

Publisher's Note: MDPI stays neutral with regard to jurisdictional claims in published maps and institutional affiliations.

Copyright: (c) 2021 by the authors. Licensee MDPI, Basel, Switzerland. This article is an open access article distributed under the terms and conditions of the Creative Commons Attribution (CC BY) license (https:// creativecommons.org/licenses/by/ $4.0 /)$.
Abstract: Recently, Streptococcus suis reference strains of serotype 20, 22, and 26 were reclassified as Streptococcus parasuis. The public health significance of $S$. parasuis is underestimated due to the lack of clinical isolates. In the present study, we first reported two sporadic S. parasuis infections in humans, after using full-length $16 \mathrm{~S}$ rRNA and housekeeping genes' phylogeny and ANI values of genome sequence comparisons to determine the species of their isolates BS26 and BS27. Compared to highly pathogenic S. suis strain P1/7, S. parasuis strains BS26 and BS27 possessed a delayed capacity to initiate lethal infection, which may attribute to the later production of higher level of proinflammatory cytokines. Differed to S. suis strain P1/7, S. parasuis strains did not induce significant inflammatory response in the brain of mice. Histopathological changes in liver and lungs were widely present in mice infected with $S$. parasuis strains. Our data indicated that the pathogenic mechanism of $S$. parasuis may be different from that of $S$. suis. Three lineages in the core-genome phylogenetic tree and ten types of cps gene cluster were found in 13 S. parasuis genomes, indicating high heterogeneity of this species. The similarity of CPS structure and antibiotic-resistant genes relative to $S$. suis indicated the evolutionary affinity between the two species. Our data suggested S. parasuis is a potential zoonotic pathogen and poses severe threat to health of susceptible people. Further study on the epidemiology and public health significance of S. parasuis is urgently necessary.

Keywords: S. parasuis; human infection; virulence; histopathologic changes; pro-inflammatory cytokines; antimicrobial resistance; phylogeny; cps

\section{Introduction}

Streptococcus suis is an important zoonotic pathogen that causes primarily meningitis, sepsis, endocarditis, arthritis, and pneumonia in both pigs and humans [1,2]. Currently, 35 serotypes (types 1 through 34 and 1/2) of $S$. suis have been identified on the basis of their capsular polysaccharide antigens (CPS) [1,3]. S. suis serotypes 32 and 34 have been reclassified as Streptococcus orisratti [4]. Recently, S. suis reference strains of serotypes 20, 22, 26 were proposed as Streptococcus parasuis [5] and serotype 33 was reclassified as Streptococcus ruminantium [6]. The presence of $S$. parasuis in diseased pigs and calves with pneumonia or systemic infection (meningitis, arthritis, endocarditis, or septicemia) indicated that $S$. parasuis may be pathogenic to pigs and/or calves [7-10]. The public health significance of $S$. parasuis has not been evaluated thoroughly, due to the lack of clinical isolates and published case reports. Strains BS26 and BS27 isolated from two patients and reported in the current study were classified as S. parasuis species by combining fulllength 16S rRNA and housekeeping genes' phylogeny and ANI values of genome sequence 
comparisons. To evaluate the potential virulence of BS26 and BS27, the survival curve, histopathological lesions, and kinetics of inducing pro-inflammatory cytokines production in vivo were compared to those of highly pathogenic S. suis strain P1/7 [11]. Additional 11 genomes of S. parasuis available in NCBI were included to investigate the phylogeny and genomic features of $S$. parasuis.

\section{Results}

2.1. Phylogenetic Analysis of Full-Length $16 S$ rRNA and Housekeeping Genes groEL, gyrB, sodA, and recN of $S$. parasuis

Strain BS27 had 16S rRNA gene sequence similarity with BS26 equal to $100 \%$. Phylogenetic analysis using 16S rRNA gene sequences demonstrated that strains BS26 and BS27 were $98.9 \%$ similar to S. parasuis type strain SUT- $286^{\mathrm{T}}$ but $96.6 \%$ similar to S. suis type strain NCTC10234 ${ }^{\mathrm{T}}$ (Table S1). A dendrogram was constructed from a similarity matrix using the $16 \mathrm{~S}$ rRNA sequences from $16 \mathrm{~S}$. parasuis strains (the full-length $16 \mathrm{~S}$ rRNA gene sequences were not extracted from draft genomes SUT-319 and SUT-380), S. suis type strain NCTC10234 ${ }^{\mathrm{T}}$, and E. faecalis JCM 5803 (Figure 1). S. suis type strain NCTC10234 ${ }^{\mathrm{T}}$ and E. faecalis JCM 5803 were distant from S. parasuis strains that fell into a group. Compared with other 14 S. parasuis strains, the similarity of $16 \mathrm{~S}$ rRNA sequences of BS26 and BS27 ranged from $98.9 \%$ to $99.6 \%$ (Table S1). The similarity of $16 \mathrm{~S}$ rRNA sequences among $16 \mathrm{~S}$. parasuis ranged from $98.8 \%$ to $100 \%$. The similarity of $16 \mathrm{~S}$ rRNA sequences between S. parasuis and S. suis ranged from $96.5 \%$ to $97.6 \%$. (Table S1).

In addition to the $16 \mathrm{~S}$ rRNA gene, housekeeping genes such as groEL, gyrB, $\operatorname{sod} A$, and $r e c N$ were also suitable and helpful for Streptococcus strain differentiation at the species level $[12,13]$. Strain BS27 shared 100\% housekeeping-gene sequence similarity with BS26. Sequence analysis of housekeeping genes groEL, gyrB, sodA, and $r e c N$ demonstrated that strains BS26 and BS27 were $97.6 \%, 97.6 \%, 97.4 \%$, and $98.7 \%$ similar to $S$. parasuis type strain SUT-286 ${ }^{\mathrm{T}}$, respectively (Table S1). Consistent with the $16 \mathrm{~S}$ rRNA gene result, S. parasuis strains were grouped together and were well separated from S. suis NCTC10234 $4^{\mathrm{T}}$ in the phylogenetic trees that were based on the groEL, gyrB, sodA, and $\operatorname{recN}$ genes (Figure 1). Divergence values of $0-5.8 \%, 0-2.8 \%, 0-2.7 \%$, and $0-3.1 \%$ among $S$. parasuis strains were obviously lower than $7-8.5 \%, 15.7-16.3 \%, 23-25 \%$, and $15.3-17 \%$ with S. suis strain NCTC10234 ${ }^{\mathrm{T}}$ for the groEL, gyrB, $\operatorname{sod} A$, and $r e c N$ genes, respectively (Table S1).

Strains BS26 and BS27 had an average nucleotide identity (ANI) value of $95.1 \%$ and $95.2 \%$ with S. parasuis type strain SUT-286 ${ }^{\mathrm{T}}$, respectively (Table S1). In contrast to the high similarity with S. parasuis type strain SUT- $286^{\mathrm{T}}$, ANI values of BS26 and BS27 were $83.42 \%$ and $83.33 \%$ with $S$. suis type strain NCTC10234 ${ }^{\mathrm{T}}$, respectively. ANI values among 13 genomes of $S$. parasuis were in the range $93.8-99.99 \%$, obviously higher than the range $83.33-84.72 \%$ with S. suis type strain NCTC10234 ${ }^{\mathrm{T}}$ (Table S1). 
A 16s rRNA

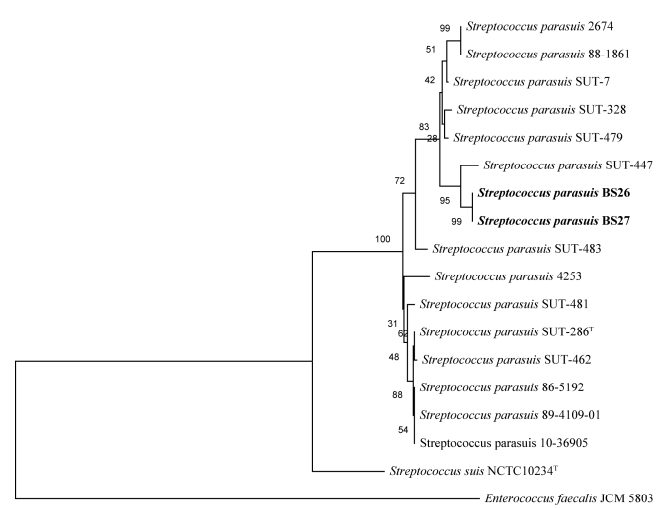

0.010

C

$\operatorname{gyr} B$

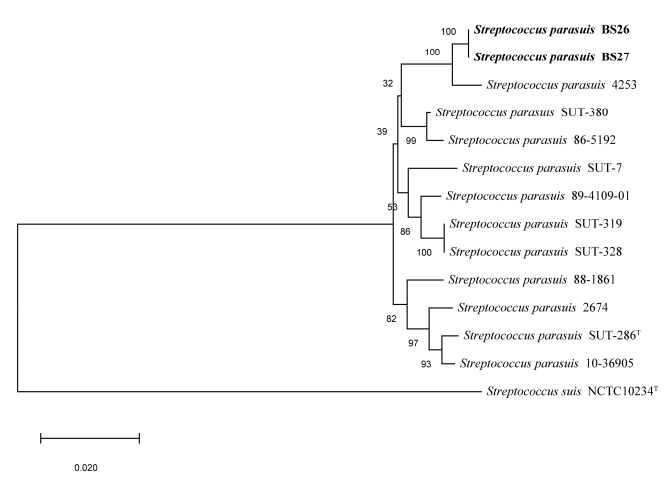

E $\operatorname{recN}$
B groEL

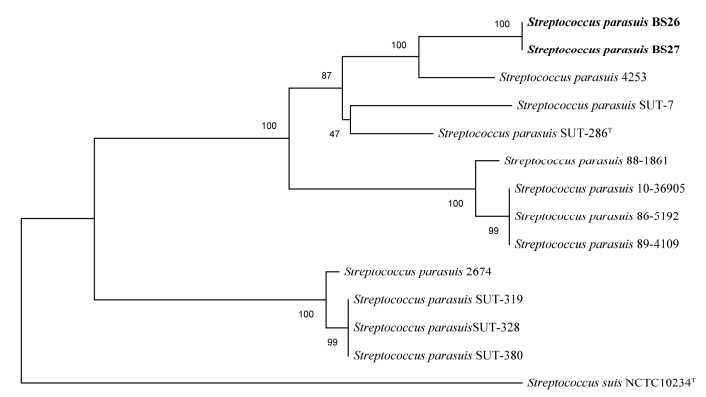

0.010

D $\operatorname{sod} A$

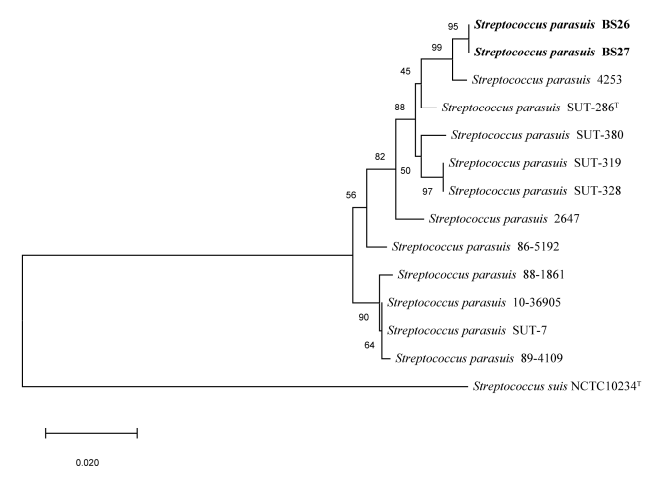

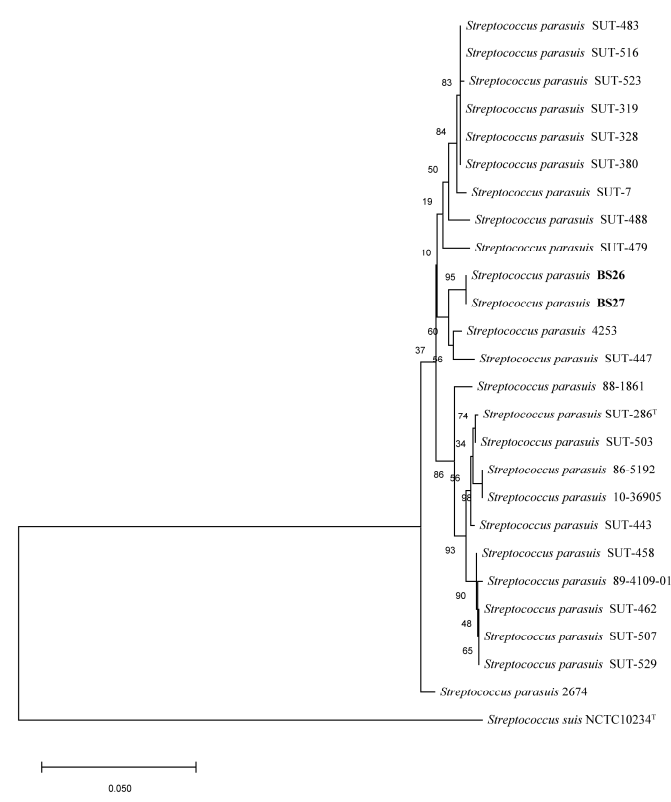

Figure 1. Phylogenetic relationships of strains used in the present study. The trees were based on an alignment of full length of the 16S rRNA (A), groEL (B), gyrB (C), sodA (D), and $\operatorname{recN}(\mathbf{E})$ by using the neighbor-joining method. The corresponding sequence of $E$. faecalis JCM 5803 (for $16 \mathrm{~S}$ rRNA gene) or S. suis type strain NCTC10234 ${ }^{\mathrm{T}}$ (for groEL, gyrB, $\operatorname{sodA}$, and recN genes) was used as an out-group to root the trees. The confidence values were obtained from 1000 replications. The bar represents sequence dissimilarity. 


\subsection{Difference in Virulence between S. suis Strain P1/7 and Two S. parasuis Strains}

In order to evaluate the potential virulence of S. parasuis BS26 and BS27, we compared the survival curve of C57BL/6 mice infected with two S. parasuis strains and highly pathogenic S. suis strain P1/7. Most mice infected with P1/7, BS26 or BS27 showed severe septic signs in period of the infection, such as rough hair coat, swollen eyes, weakness, and shivering.

The survival rate of mice infected with S. suis strain P1/7 or two S. parasuis strains was significantly different from that of mock-infected mice. Moreover, significant difference in the survival level was also observed between mice infected with S. suis strain P1/7 and two S. parasuis strains, which attributed to the differences at an early phase of the infection. Mice infected with S. suis strain P1/7 had a $20 \%$ survival rate at $8 \mathrm{~h}$ post-infection, while mice infected with S. parasuis strain BS26 and BS27 had a 95\% and 90\% survival rate at the same time point, respectively. It is noteworthy that the survival levels of mice infected with BS26 and BS27 dramatically decreased after $12 \mathrm{~h}$ post-infection. At $24 \mathrm{~h}$ postinfection, survival rates of the P1/7-infected group (15\%), BS26-infected group (20\%), and BS27-infected group (25\%) were very similar (Figure 2 and Table S2).

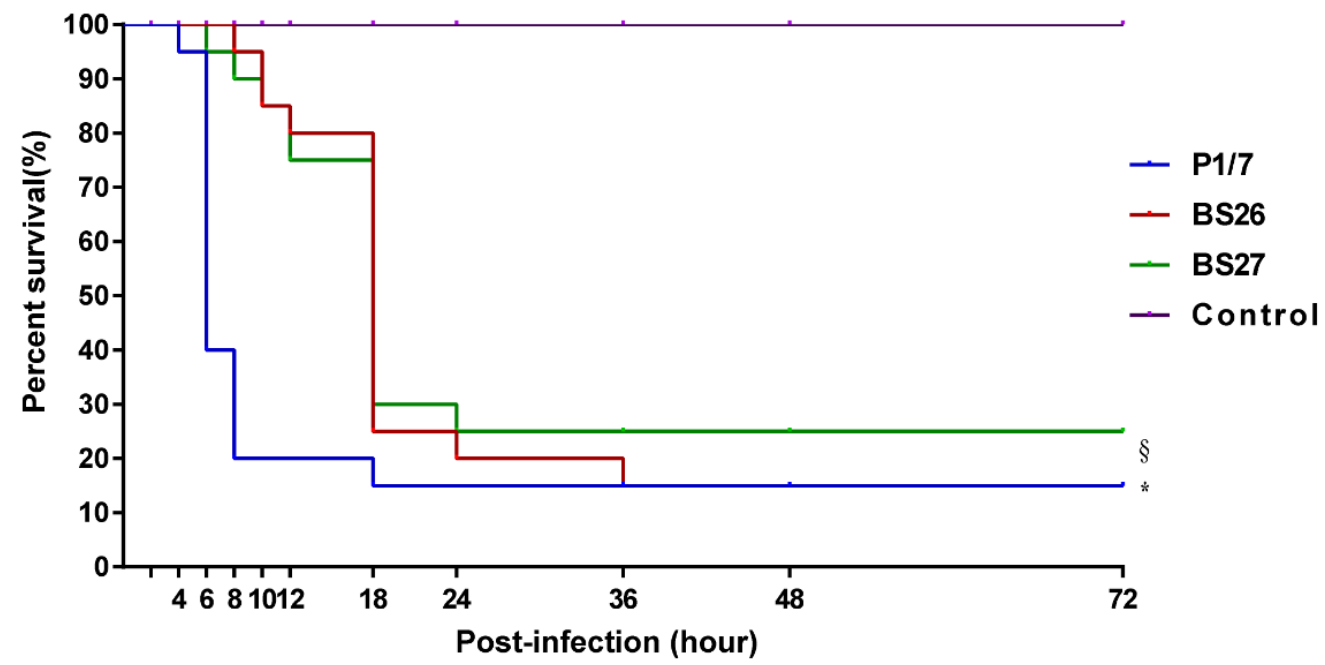

Figure 2. Survival curves of mice injected with $5 \times 10^{7} \mathrm{CFU}$ of live S. parasuis strain BS26, S. parasuis strain BS27, S. suis strain P1/7, and PBS only as control group. Overall survival rates of the two experiments for each group (calculated via the Kaplan-Meier method) at $2 \mathrm{~h}, 4 \mathrm{~h}, 6 \mathrm{~h}, 8 \mathrm{~h}, 10 \mathrm{~h}, 12 \mathrm{~h}$, $18 \mathrm{~h}, 24 \mathrm{~h}, 36 \mathrm{~h}, 48 \mathrm{~h}$, and $72 \mathrm{~h}$ post-infection were present in survival curve. The survival rates of different groups were compared using Log-rank test. *, significantly different $(p<0.05)$ compared to $S$. parasuis strains-infected group and control group. $\S$, significantly different $(p<0.05)$ between S. parasuis strains-infected group and control group.

\subsection{Histopathological Lesions and Bacterial Load in Survival Mice}

Totally, three mice infected with $S$. suis strain $\mathrm{P} 1 / 7$, three mice infected with $S$. parasuis strain BS26, and five mice infected with $S$. parasuis strain BS27 survived at $72 \mathrm{~h}$ postinfection in survival experiment. Strains were isolated in the peripheral blood, brain, lung, liver, spleen, and kidney of all survival mice.

Slight deformation of neuron was found in the brains of seven mice infected with S. parasuis strain (Figure 3A(1)). Only one mouse infected with S. parasuis BS26 was observed slightly infiltrated by lymphocytes (Figure 3A(1)). Histopathological lesions of S. parasuis-infected mice were mainly observed in the lung and liver. These organs presented bacterial loads over $10^{2} \mathrm{CFU} / 0.1 \mathrm{~g}$. Hepatocyte steatosis was significant histopathological changes of livers and was present in all S. parasuis-infected mice (Figure 3B(1)). Coagulative necrosis was observed in the liver of two mice infected with the S. parasuis strain (Figure $3 \mathrm{~B}(1)$ ). Alveoli wall thickening with diffused infiltration of neutrophils and lymphocytes was observed in the lung of all S. parasuis-infected mice (Figure 3C(1)). Some 
hemorrhagic foci in lungs were also found in the two mice infected with S. parasuis strain (Figure 3C(2)). No significantly histopathological changes were observed in the heart and spleen of $S$. parasuis-infected mice.

A1

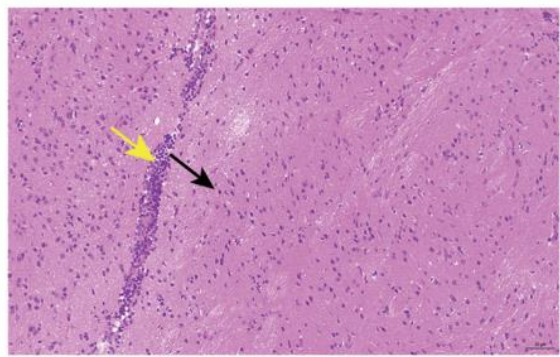

B1

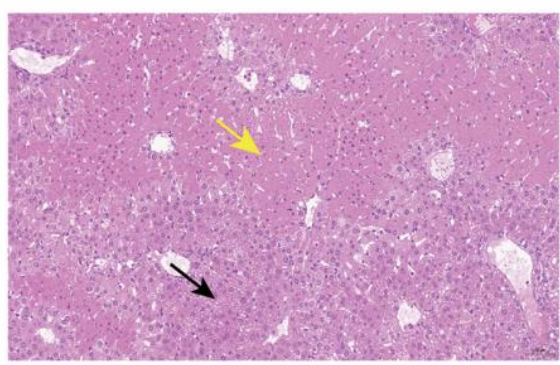

C1

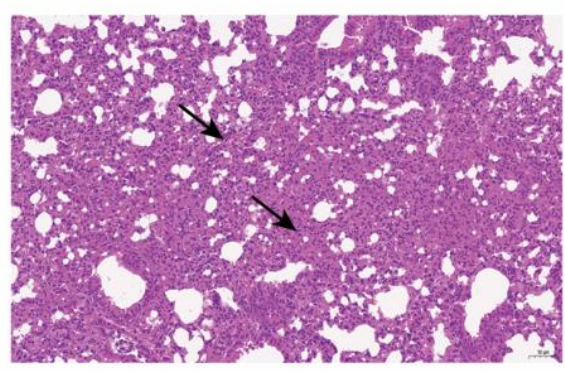

$\mathrm{C} 3$

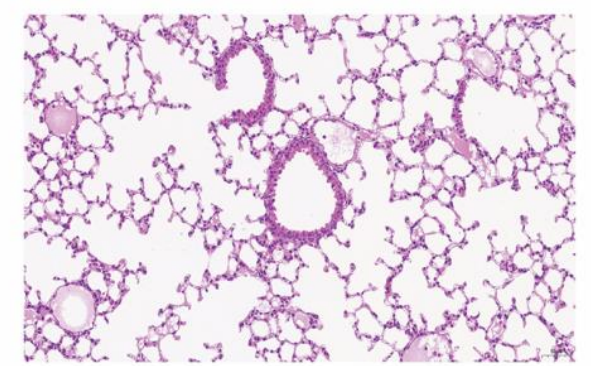

A2

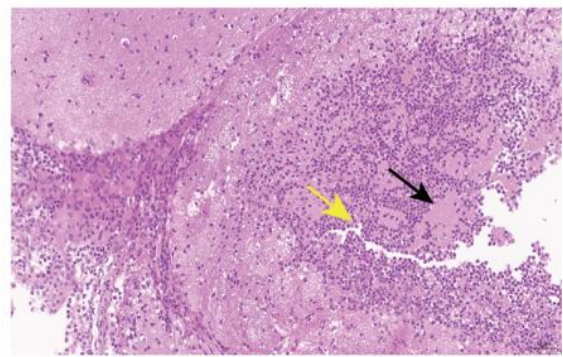

B2

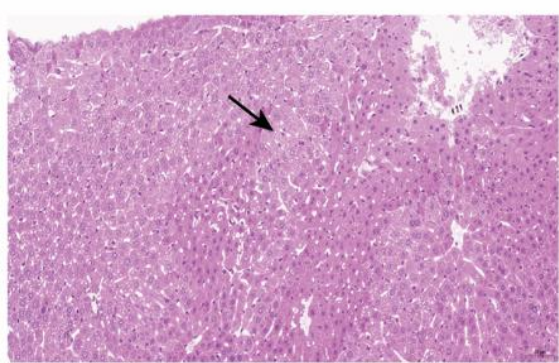

$\mathrm{C} 2$

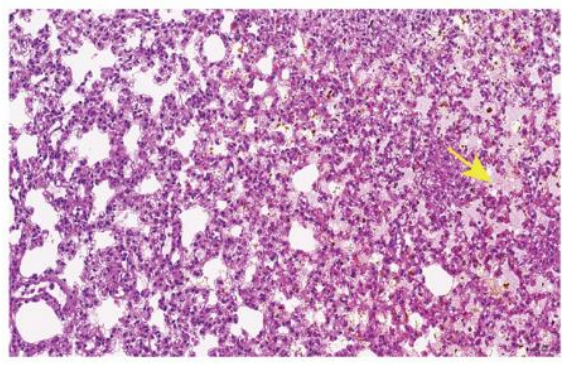

$\mathrm{D}$

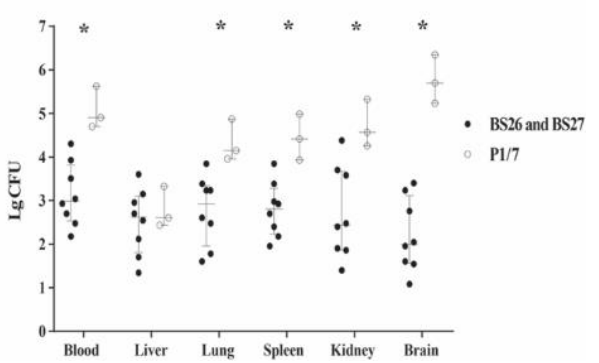

Figure 3. Histopathological changes and bacterial loads in the organs of survival mice in survival experiment. (A1) Micrograph of brain sample from a S. parasuis strain BS26-infected mouse at $72 \mathrm{~h}$ post-infection. Neuronal deformation (black arrowhead) and slight infiltration by lymphocytes (yellow arrowhead) were shown. (A2) Micrograph of brain sample from a mouse infected with S. suis strain P1/7 at $72 \mathrm{~h}$ post-infection. Neuronal necrolysis (black arrowhead) and significant infiltration 
by neutrophils (yellow arrowhead) were shown. (B1) Micrograph of liver sample from a mouse infected with S. parasuis strain BS26 at $72 \mathrm{~h}$ post-infection. Hepatocyte steatosis (black arrowhead) and coagulative necrosis (yellow arrowhead) were shown. (B2) Micrograph of liver sample from a mouse infected with $S$. suis strain P1/7 at $72 \mathrm{~h}$ post-infection. Edema of few hepatocytes was shown (black arrowhead). (C1) Micrograph of lung sample from a mouse infected with S. parasuis strain BS26 at $72 \mathrm{~h}$ post-infection. Alveoli wall thickening with diffused infiltration of neutrophils and lymphocytes (black arrowhead) was shown. (C2) Micrograph of lung sample from a mouse infected with S. parasuis strain BS26 at $72 \mathrm{~h}$ post-infection. Hemorrhagic foci (yellow arrowhead) are shown. (C3) Micrograph of lung sample from a mouse infected with S. suis strain P1/7 at $72 \mathrm{~h}$ postinfection. No significant histopathological lesions were observed. H\&E staining, $\times 200$ magnification, scale: $50 \mu \mathrm{m}$. (D) Bacterial loads in blood and organs of survival mice. Colonies were expressed as CFU/0.1 $\mathrm{g}$ for organ samples or CFU/mL for blood samples. Bacterial counts of individuals, including median with interquartile ranges, are presented. Significant difference in bacterial counts between $S$. suis and S. parasuis-infected groups was determined by Wilcoxon's two-sample test. *, significantly different $(p<0.05)$ in bacterial counts between $S$. suis strain P1/7-infected group and $S$. parasuis strains-infected group.

Different from S. parasuis strains-infected mice, neuronal necrolysis and significant infiltration by neutrophils were observed in brains of all mice infected with $S$. suis strain P1/7 (Figure $3 \mathrm{~A}(2)$ ). Although bacterial loads ranged from $10^{2}$ to $10^{4} \mathrm{CFU} / 0.1 \mathrm{~g}$ in other organs of mice infected with $S$. suis strain P1/7, no significantly histopathological lesions were observed in them (Figure $3 \mathrm{~B}(2), \mathrm{C}(3)$ ). Bacterial counts of mice infected with $S$. suis were significantly higher than those of mice infected with $S$. parasuis, except for the liver (Figure 3D).

2.4. Pro-Inflammatory Cytokine Production and Bacterial Loads in Mice Infected with S. parasuis Strain BS26 and S. parasuis Strain P1/7

Proinflammatory cytokines were responsible for the death of mice infected with $S$. suis strains within $24 \mathrm{~h}$ post infection [14-16]. The kinetics of proinflammatory cytokines induced by P1/7 and BS26 within $24 \mathrm{~h}$ post-infection was compared. Stimulations with S. parasuis strain BS26 and S. suis strain P1/7 induced time-dependent production of IL-6 and TNF-a in mice. The kinetics and levels of IL-6 and TNF-a that were induced in vivo were different between the two strains. S. suis strain P1/7 was able to induce significantly more rapid production and higher levels of IL-6 and TNF-a early in the post-infection period, reaching peaks of 54,027 pg/mL and $2067 \mathrm{pg} / \mathrm{mL}$ at $8 \mathrm{~h}$, respectively. Then, levels of cytokines gradually decreased from $12 \mathrm{~h}$ to $24 \mathrm{~h}$ post-infection. The pattern of TNFa induced by BS26 in vivo was similar to those patterns of cytokines induced by P1/7, peaking at $8 \mathrm{~h}$ post-infection and gradually decreasing thereafter. In contrast, the level of IL-6 induced in vivo by BS26 peaked at 34,690 pg/mL at $12 \mathrm{~h}$ post-infection and gradually decreased thereafter. Moreover, BS26 induced higher levels of IL- 6 than P1/7 did at $12 \mathrm{~h}$ post-infection. Significantly higher levels of cytokines were produced by $S$. suis strain $\mathrm{P} 1 / 7$ at $4,8,16$, and $24 \mathrm{~h}$ post-infection than those produced by $S$. parasuis strain BS26 (Figure 4A,B).

In the present study, we also counted the bacterial loads in the peripheral blood, liver, lung, and brain of infected mice at $4,8,12,16$, and $24 \mathrm{~h}$ post-infection. The bacterial counts in the peripheral blood and organs of mice infected with $S$. suis strain P1/7 were statistically higher than those of S. parasuis strain BS26-infected mice.

In $S$. parasuis strain BS26-infected group, bacterial counts in the peripheral blood reached $10^{6} \mathrm{CFU} / \mathrm{mL}$ at $4 \mathrm{~h}$ post-infection; and then descended dramatically to $10^{4}$ $\mathrm{CFU} / \mathrm{mL}$ at 8 and $12 \mathrm{~h}$ post-infection, and $10^{2} \mathrm{CFU} / \mathrm{mL}$ at $24 \mathrm{~h}$ post-infection (Figure $4 \mathrm{C}$ ). Viable counts in the brain presented similar kinetics, peaked at $10^{4} \mathrm{CFU} / 0.1 \mathrm{~g}$ at $4 \mathrm{~h}$ postinfection and gradually decreased to $10^{3} \mathrm{CFU} / 0.1 \mathrm{~g}$ at $24 \mathrm{~h}$ post-infection (Figure 4D). In contrast, bacterial counts in the liver and lung showed a different pattern. Viable counts in the liver and lung reached approximately $10^{6} \mathrm{CFU} / \mathrm{mL}$ at $4 \mathrm{~h}$ post-infection, peaked 
over $10^{6} \mathrm{CFU} / 0.1 \mathrm{~g}$ at $12 \mathrm{~h}$ post-infection and gradually decreased to $10^{5} \mathrm{CFU} / 0.1 \mathrm{~g}$ at $24 \mathrm{~h}$ post-infection (Figure $4 \mathrm{E}, \mathrm{F}$ ). It is noteworthy that bacterial counts in the liver and lung were higher than that of peripheral blood from $8 \mathrm{~h}$ to $24 \mathrm{~h}$ post-infection.

A

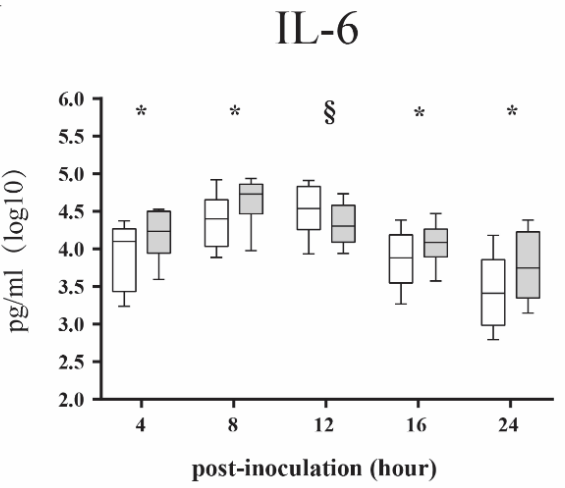

$\mathrm{C}$

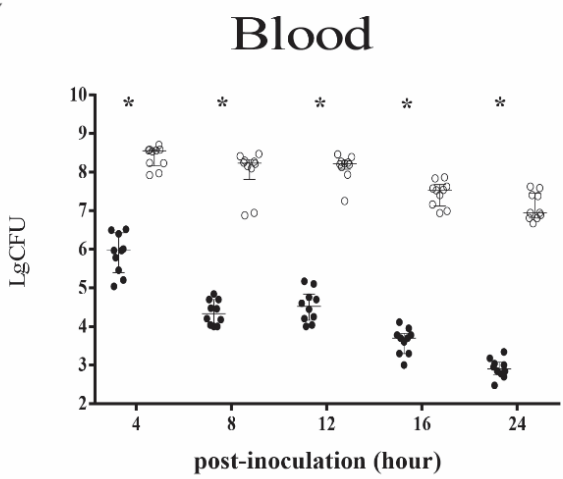

$\mathrm{E}$

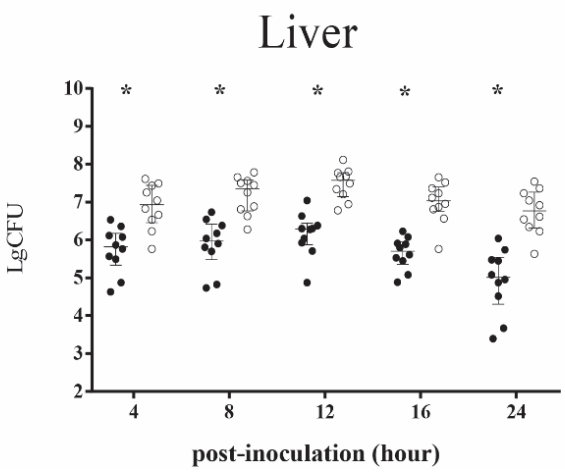

B

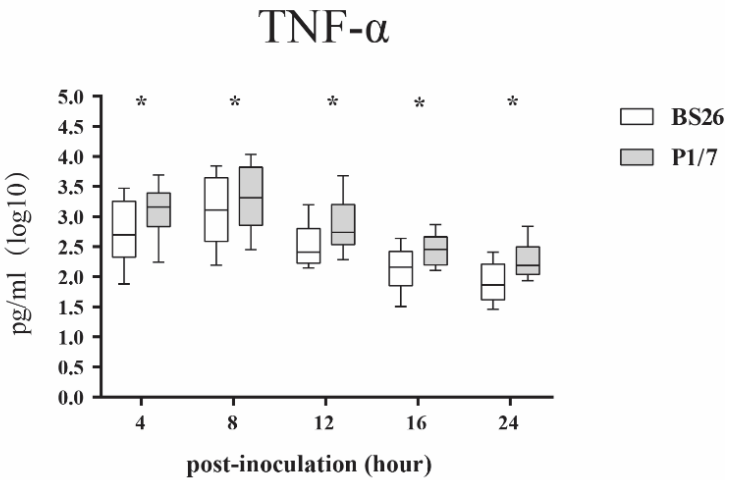

$\mathrm{D}$

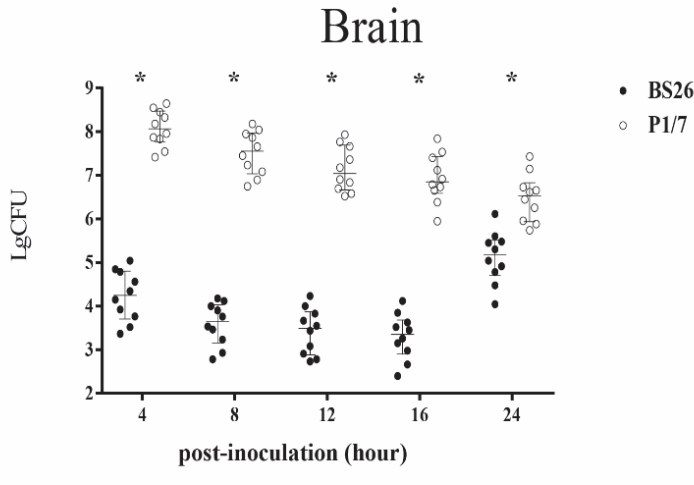

F

- BS26 - $\mathbf{P 1 / 7}$

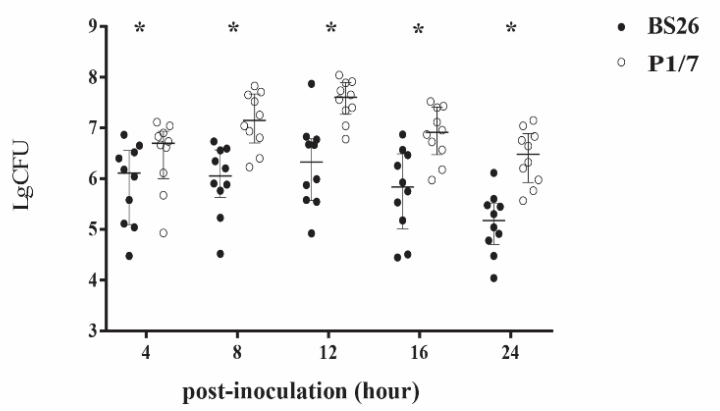

Figure 4. Production of pro-inflammatory cytokines IL-6 (A) and TNF- $\alpha$ (B) in sera and bacterial loads (C-F) of C57BL/6 mice infected with $1 \times 10^{7} \mathrm{CFU}$ of S. suis strain P1/7 and S. parasuis strain BS26. Median values with interquartile ranges of each group were used to express cytokine levels in sera. Bacterial counts of individuals, including median with interquartile ranges, were presented. Colonies were expressed as $\mathrm{CFU} / 0.1 \mathrm{~g}$ for organ samples or $\mathrm{CFU} / \mathrm{mL}$ for blood samples. Significant differences in bacterial counts and cytokine data between the two infected groups were determined by Wilcoxon's twosample test. * Cytokine levels and bacterial counts of mice infected with $S$. suis strain P1/7 were statistically higher than those of mice infected with S. parasuis strain BS26 $(p<0.05)$. § Cytokine levels of mice infected with S. parasuis strain BS26 were statistically higher than those of mice infected with S. suis strain P1/7 $(p<0.05)$.

In S. suis strain P1/7-infected group, bacterial counts in the peripheral blood reached $10^{8} \mathrm{CFU} / \mathrm{mL}$ at $4 \mathrm{~h}$ post-infection, remained at $10^{8} \mathrm{CFU} / \mathrm{mL}$ at 8 and $12 \mathrm{~h}$ post-infection, and slightly decreased to $10^{6} \mathrm{CFU} / \mathrm{mL}$ at $24 \mathrm{~h}$ post-infection (Figure $4 \mathrm{C}$ ). Kinetics of viable counts in the organs were similar to those of mice infected with S. parasuis strain BS26. Viable counts in the brain peaked at $10^{8} \mathrm{CFU} / \mathrm{mL}$ at $4 \mathrm{~h}$ post-infection and gradually decreased to $10^{6} \mathrm{CFU} / \mathrm{mL}$ at $24 \mathrm{~h}$ post-infection (Figure $4 \mathrm{D}$ ). Viable counts in the liver 
and lung reached $10^{6} \mathrm{CFU} / 0.1 \mathrm{~g}$ at $4 \mathrm{~h}$ post-infection, peaked at $10^{7} \mathrm{CFU} / 0.1 \mathrm{~g}$ at $12 \mathrm{~h}$ post-infection, and slightly decreased to $10^{6} \mathrm{CFU} / 0.1 \mathrm{~g}$ at $24 \mathrm{~h}$ post-infection (Figure $4 \mathrm{E}, \mathrm{F}$ ).

\subsection{AR Genes and Antimicrobial Susceptibility Profiles of BS26 and BS27}

Only $m s r(D)$ and $m e f(A)$ genes that code for resistance to macrolides were found in genomes of BS26 and BS27. Lower levels of resistance to erythromycin and azithromycin was found in S. parasuis strains BS26 and BS27, and those levels were attributed to $m s r(D)$ and $\operatorname{mef}(A)$ genes with MICs 8 and $24 \mu \mathrm{g} / \mathrm{mL}$, respectively. It is noteworthy that $S$. parasuis strains BS26 and BS27 were resistant to trimethoprim-sulfamethoxazole with an MIC $>32 \mu \mathrm{g} / \mathrm{mL}$, although they did not harbor AR genes that were known to be related. In addition, S. parasuis strain BS26 and BS27 were susceptible to penicillin G, cefaclor, clindamycin, vancomycin, chloramphenicol, tetracycline, streptomycin, kanamycin, spectinomycin, and gentamicin.

\subsection{Comparative Genomic Analysis of S. parasuis}

The core-genome phylogenetic tree based on 442 core genes of 14 Streptococcus genomes indicated great diversity among $S$. parasuis genomes, which were clustered into three discrete lineages (Figure 5). Lineage 1 contained BS26, BS27, and four additional public genomes. Lineage 2 contained the reference genomes of serotype 20, 22, and 26, type strain SUT- $286^{\mathrm{T}}$, and two additional public genomes. Lineage 3 only contained SUT-7, which was distant from other S. parasuis genomes.

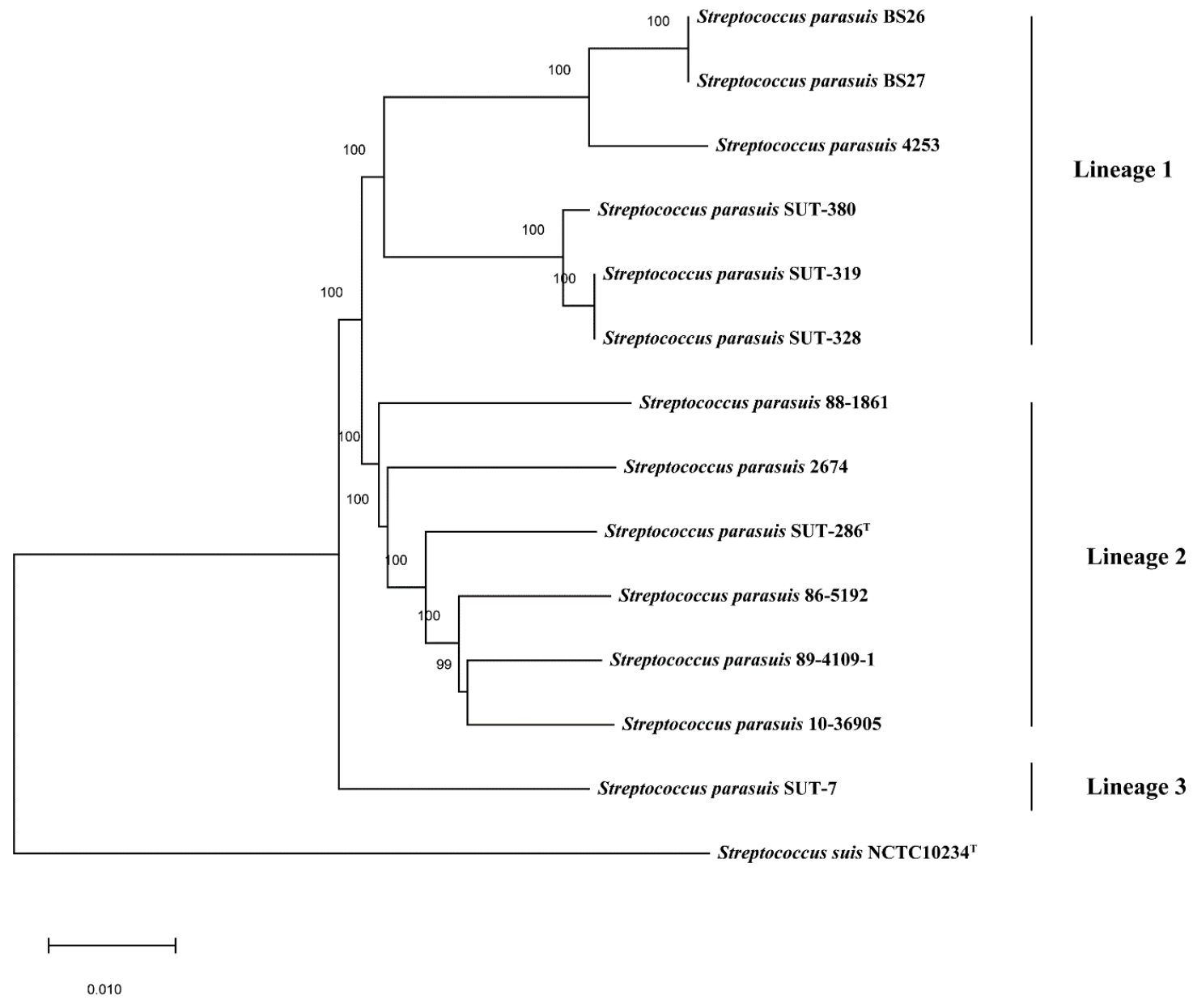

Figure 5. Core-genome phylogeny of 14 Streptococcus genomes in the present study. The tree was based on an alignment of 442 core genes of 13 S. parasuis genomes and 1 S. suis genome using the neighbor-joining method. The S. suis type strain NCTC10234 ${ }^{\mathrm{T}}$ was used as an out-group to root the tree. The bar represents sequence dissimilarity. 


\subsection{The Analysis of Cps Gene Clusters of S. parasuis}

Complete $c p s$ gene clusters were extracted from eleven of $13 \mathrm{~S}$. parasuis genomes. The three sides of cps gene clusters was deleted in genomes SUT-286 ${ }^{\mathrm{T}}$ and 4253 . The cps gene cluster of BS26 was identical to that of BS27. The cps gene clusters of S. parasuis were located between the CDS1210 and CDS1209 genes (named as homologous genes in S. suis genome P1/7) and belonged to pattern III, identical to that of reference strains of serotype 20,22, and 26 [17]. The fact that all cps gene clusters of S. parasuis harbored $w z y / w z x$ genes indicated that CPs of $S$. parasuis were synthesized by the WZX/WZY pathway. The conserved $\operatorname{cps} A$, $c p s B, c p s C$, and $c p s D$ were present and located on the $5^{\prime}$ side of the $c p s$ gene clusters. The central regions of these clusters were highly variable. The $c p s$ gene clusters were clustered into ten types (I to X) based on the sequence of the wzy gene (Table 1). Among them, cps types VI and X contained three genomes (SUT-319, SUT-328, and SUT-380) and two genomes (BS26 and BS27), respectively. Other cps types comprised one genome each. The wzy genes of type V, VII, IX, and X were highly homologous to those of S. thermophilus EPS type VI, S. suis serotype 24 , S. suis serotype 10 , and S. thermophilus EPS type V, respectively (Figure 6).

Table 1. The information on S. parasuis genomes used in the study.

\begin{tabular}{cccccc}
\hline Strains & Source & Location & Year & cps Type & Accession No. \\
\hline BS26 & Patient & China & 2018 & X & CP069079 \\
BS27 & Patient & China & 2018 & X & JAETXU000000000 \\
$86-5192$ & Diseased calf & United States & $1980^{\prime}$ & I & PRJNA171426 \\
$88-1861$ & Diseased pig & Canada & $1980^{\prime}$ & II & PRJNA171444 \\
$89-4109-1$ & Diseased pig & $/$ & $1980^{\prime}$ & III & PRJNA171433 \\
SUT-7 & Healthy pig & Japan & $/$ & V & DRX016751 \\
SUT-286 & Healthy pig & Japan & $/$ & IV & DRX016752 \\
SUT-319 & Healthy pig & Japan & $/$ & VI & DRX016753 \\
SUT-328 & Healthy pig & Japan & $/$ & VI & DRX016754 \\
SUT-380 & Healthy pig & Japan & $/$ & VI & DRX016755 \\
$10-36905$ & Healthy Bos taurus & United States & 2010 & VII & PRJNA590796 \\
2674 & Healthy pig & China & 2014 & VIII & POIG00000000 \\
4253 & Healthy cow & Switzerland & 2018 & IX & SHGT00000000 \\
\hline
\end{tabular}




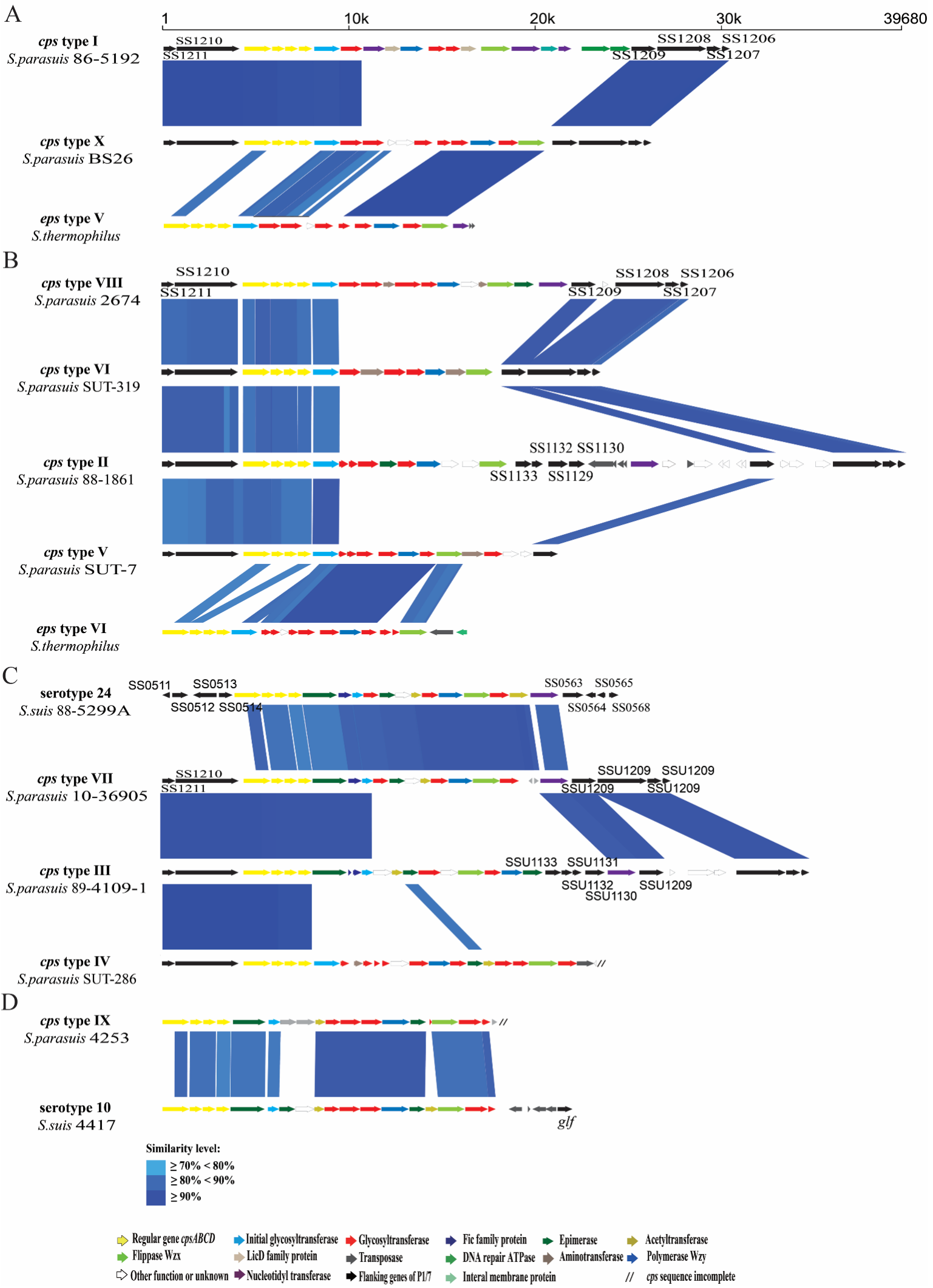

Figure 6. Schematic comparison of the cps gene loci among (A) S. parasuis type I, X, and S. thermophilus strain type V. (B) S. parasuis type II, V, VI, VIII, and S. thermophilus strain type VI. (C) S. parasuis type III, IV type VII, and S. suis serotype 24. (D) S. parasuis type IX, and S. suis serotype 10. Each colored arrow represents a gene whose predicted function is shown in the blown-up panel. The direction of the arrow indicates the direction of transcription. Regions of $70 \%$ identity were marked by blue shading. 


\section{Discussion}

Two strains-BS26 and BS27-were isolated from the blood cultures of two patients and initially identified as $S$. suis by MALDI-TOF MS. In the present study, the $16 \mathrm{~S}$ rRNA gene sequence similarity between two strains and S. parasuis type strain SUT- $286^{\mathrm{T}}$ exceeded $98.7 \%$, the threshold value to delineate the species [18]. Sequence analysis of their groEL, gyrB, rpoB, sod $A$, and recN genes also showed that the two strains had phylogenetic affinity with the $S$. parasuis species.

ANI value can be used as a parameter to determine whether two genomes belong to the same species. The ANI cut-off value for species definition is species-dependent. Average ANI of the same species varies from 0.779 (Polynucleobacter necessaries) to 0.998 (Leptospira biflexa) [19]. By comparison of pairs of 1226 bacterial strains with whole genome sequences, bacterial species definition using cut-off value of 0.92 matched the current bacterial species definition well [19]. In the present study, BS26 and BS27 had ANI values of $95.1 \%$ and $95.2 \%$ with $S$. parasuis type strain SUT- $286^{\mathrm{T}}$, respectively. These ANI values are higher than the 95\% cut-off ANI value for bacterial species that Goris et al. proposed [20]. It is important that full-length $16 \mathrm{~S}$ rRNA and housekeeping genes' and core-genome phylogeny demonstrated that $S$. suis type strain $\mathrm{NCTC} 10234^{\mathrm{T}}$ formed a distinct branch and was well separated from S. parasuis. By combining full-length $16 \mathrm{~S}$ rRNA and housekeeping genes' phylogeny and ANI values of genome sequence comparisons, it is concluded that the BS26 and BS27 strains belonged to the S. parasuis species. Before the present study, S. parasuis had been isolated from healthy and diseased pigs or calves [7-10]. The present study makes the first report of sporadic $S$. parasuis infections in humans. It is possible that the prevalence and clinical relevance of $S$. parasuis have been underestimated because of a lack of effective methods to identify it.

To evaluate the potential virulence of S. parasuis strains BS26 and BS27, the survival curve, histopathological lesions, and kinetics of pro-inflammatory cytokines production in vivo were compared to those of highly pathogenic S. suis strain P1/7. Compared to S. parasuis strain BS26- and BS27-infected mice, those infected with S. suis strain P1/7 quite rapidly succumbed to the infection. Significant differences at the early phase of infection and similarity at the middle phase of infection in survival level were observed between $S$. suis strain P1/7-infected group and S. parasuis strains-infected groups. The results indicated that $S$. parasuis strains BS26 and BS27 possessed a delayed capacity to initiate lethal infection.

The histopathological lesions in major organs were also investigated in all survival mice at $72 \mathrm{~h}$ post infection of survival assay. Consistent with the results of previous study [15], survival mice infected with $S$. suis strain P1/7 initiated significant inflammation in brains. Obviously higher bacterial loads present in brains of mice infected with S. suis P1/7 may contribute to the histopathological changes. No significant inflammatory response was found in the brains of the survival mice infected with $S$. parasuis strains. Histopathological lesions of survival mice infected with $S$. parasuis were widely present in lungs and livers. Hepatocyte steatosis was present in all S. parasuis infected mice. Hepatic mitochondrial and its enzyme activity may be impaired in response to sepsis induced by S. parasuis, causing hepatic lipid utilization disorder. Significant inflammation in lungs were observed in all survival mice infected with the $S$. parasuis strain. It is noteworthy that S. parasuis strain BS27 also led to the inflammation of both lungs in its host.

In previous studies, no significant histopathological changes in organs were observed in dead mice infected with $S$. suis within $48 \mathrm{~h}$ post-infection [14,15]. Proinflammatory cytokines played a critical role in the death of mice or patients infected with $S$. suis within $24 \mathrm{~h}$ post-infection $[15,16,21]$. The overproduction of pro-inflammatory cytokines such as TNF-a and IL-6 plays an important role in the infection caused by S. suis [21]. IL-6 is an important inducer of acute phase proteins [22]. High levels of IL-6 correlate inversely with survival time in patients with sepsis [23]. TNF- $\alpha$ is one of the most important host mediators in the pathogenesis of septic shock [24] and may be responsible for the streptococcal toxic shock-like syndrome (STSLS) observed in the Sichuan outbreak [25]. 
In order to explicate the difference in mortality between mice infected with $S$. suis strain P1/7 and S. parasuis strains at early phase of infection, we also compared their kinetics of inducing TNF-a and IL-6 production in vivo within $24 \mathrm{~h}$ post-infection. The capacity of $S$. parasuis strain BS26 to induce TNF- $\alpha$ production in vivo is obviously lower than that of S. suis strain P1/7 during the whole infection. However, S. parasuis BS26 induced higher levels of IL-6 at $12 \mathrm{~h}$ post-infection. Interestingly, the survival levels of mice infected with S. parasuis srains dramatically decreased after $12 \mathrm{~h}$ post-infection. The later inflammatory response might result in most mice infected with $S$. parasuis strains succumbed to the infection at middle stage of survival experiment.

It is noteworthy that the bacterial loads of S. parasuis strain BS26 in peripheral blood and major organs were obviously lower than those of $S$. suis strain P1/7 during the infection. Moreover, S. parasuis strains BS26 and BS27 did not harbor "classical" S. suis virulence markers mrp, sly, and epf (data were not shown). Combined with the result of histopathology, we suspected the pathogenic mechanism of $S$. parasuis may be different from that of $S$. suis.

In the present study, both $S$. parasuis strains carried AR genes $m s r(D)$ and $m e f(A)$ that are mainly associated with resistance to macrolide. The category and number of AR genes carried by S. parasuis strains BS26 and BS27 were lower than those of S. suis clinical strains [26,27]. The acquisition and dissemination of AR genes in $S$. suis are strongly associated with mobile genetic elements (MGEs), which are mainly integrative and conjugative elements (ICEs) and prophages [28]. We did not find MGEs in 13 genomes of $S$. parasuis (data were not shown). Further studies are needed to investigate the formation and dissemination mechanisms of AR genes in S. parasuis population.

In the present study, the phylogeny of 13 S. parasuis genomes were analyzed. The ANI values range from $93.8 \%$ to $99.99 \%$ indicating obvious heterogeneity among S. parasuis genomes. Consistent with the ANI values, the core-genome phylogeny clustered 13 S. parasuis genomes into three discrete lineages. S. parasuis strains BS26 and BS27 had phylogenic affinity with $S$. parasuis strain 4253 and were clustered into Lineage 1, which also contained three other genomes: SUT-319, SUT-328, and SUT-380.

In the present study, the genetic characteristics of cps loci in each genome were investigated. As with that of reference strains of serotypes 20, 22, and 26, all complete cps gene clusters of $S$. parasuis belonged to pattern III [17]. They shared highly conserved $c p s A, c p s B, c p s C$, and $c p s D$ genes and flanked regions with serotype reference strains of $S$. suis, indicating the phylogenetic affinity between $S$. suis and $S$. parasuis. The possession of $w z y / w z x$ genes in each cps gene cluster strongly suggests that the CPs of all S. parasuis were synthesized using the WZX/WZY pathway. The cps gene clusters were clustered into ten groups on the basis of the sequences of $w z y$ gene. In a previous study, SUT$286^{\mathrm{T}}$ and SUT-380 agglutinated with the antisera of serotypes 20 and 22, respectively, whereas isolates SUT-7, SUT-319, and SUT-328 cross-reacted with antisera 22/26, 20/22, and 20/22, respectively [5]. However, obvious differences in sequences of the central regions including $w z x$ and wzy genes were found among them in the present study. We reasonably speculate that the agglutination reactions were non-specific. Moreover, the central regions including $w z x$ and $w z y$ genes of four cps types of $S$. parasuis had high similarity with those of $S$. thermophilus and $S$. suis, respectively. Previous studies have shown recombination between cps gene clusters of $S$. suis and S. pneumonia $[29,30]$. These data indicated that intraspecies horizontal transfer of cps gene clusters among Streptococcus had frequently occurred. The presence of many different types of cps loci in S. parasuis also indicated high heterogeneity of this species.

In conclusion, we first reported the sporadic S. parasuis infections in humans. Two S. parasuis strains isolated in patients possessed a delayed capacity to initiate lethal infection. Our data suggested $S$. parasuis as a potentially zoonotic pathogen could severely threat to health of susceptible people. Obvious heterogeneity was found among S. parasuis genomes. The similarity of CPS structure and AR genes relative to S. suis indicated the evolutionary 
affinity between the two species. Further study on the epidemiology and public health significance of $S$. parasuis is urgently necessary.

\section{Materials and Methods}

\subsection{Bacterial Strains and Case Description}

Strains BS26 and BS27 were isolated from blood cultures of peritonitis patient and arthritis patient with pneumonia in 2018, respectively. They were identified as S. suis by matrix-assisted laser desorption/ionization time-of-flight (MALDI-TOF, Microflex LRF, Bruker) method with log-score 1.82 and $1.85(1.700<$ score $<1.999$ indicates "probable genus identification"), respectively. The two strains were confirmed as not belonging to $S$. suis by amplifying a nearly complete $16 \mathrm{~S}$ rRNA gene using primer $27 \mathrm{~F}\left(5^{\prime}-\right.$ AGAGTTTGATCMTGGCTCAG- $3^{\prime \prime}$ ) and 1492R (5'-TACGGYTACCTTGTTACGACTT-3') and S. suis-specific recN and gdh genes [31-34]. Data of 16S rRNA sequencing and the PCR method detecting $r e c N$ gene specific to $S$. parasuis [6] indicated that the two strains belonged to the $S$. parasuis species.

On 9 June 2018, a 27-year-old female patient as host of BS26 was admitted to the Affiliated Hospital of Youjiang Medical University for Nationalities in Baise city because of peritonitis characterized by acute onset of fever (body temperature of $38.1^{\circ} \mathrm{C}$ ) and persistent periumbilical colic. Two years earlier, the patient was diagnosed with renal hypertension. The serum level of high-sensitivity C-reactive protein (hsCRP) (cobas c 702, Roche) measured by nephelometry and total counts of white blood cells (WBC) (Automated Hematology Analyzer XN series XN-20, sysmex) measured by flow cytometry were $31.11 \mathrm{mg} / \mathrm{L}$ and $10.2 \times 10^{9} / \mathrm{L}$, respectively. The patient's blood pressure was $164 / 101 \mathrm{~mm}$ Hg. Ceftazidime and cefazolin sodium pentahydrate were introduced by antibiotic therapy. The patient recovered and was discharged.

On 8 October 2018, a 53-year-old male patient as host of BS27 was admitted to the same hospital (as was the host of BS26) because of the acute onset of fever (body temperature of $38.9^{\circ} \mathrm{C}$ ), chill, cough, expectoration, and persistent pain in the right knee joint. His previous health status was well. The patient had been exposed to pigs one week prior to the onset of illness. A CT scan image indicated inflammation of both lungs. The serum level of hsCRP (cobas c 702, Roche) measured by nephelometry and total counts of WBC (Automated Hematology Analyzer XN series XN-20, sysmex) measured by flow cytometry were $54.84 \mathrm{mg} / \mathrm{L}$ and $22 \times 10^{9} / \mathrm{L}$, respectively. Cefatriaxone was introduced for two weeks. The patient recovered.

\subsection{DNA Extraction, Sequencing, and Bioinformatic Analysis}

The strains were grown overnight on Columbia blood base agar (Thermo Fisher Scientific, Beijing, China) at $37{ }^{\circ} \mathrm{C}$ with $5 \% \mathrm{CO}_{2}$. The genomic DNA of two strains was extracted and purified with the Wizard Genomic DNA Purification kit (Promega, Madison, WI, USA). A complete genome of BS26 was sequenced using PacBio Sequel platform and Illumina NovaSeq PE150. A draft genome of BS27 was sequenced using Illumina NovaSeq PE150. PacBio Sequel platform Libraries for single-molecule real-time (SMRT) sequencing was constructed with an insert size of $10 \mathrm{~kb}$ using the SMRT bell TM Template kit, version 1.0. In summary, the process was to fragment and concentrate DNA, repair DNA damage and ends, prepare blunt ligation reaction, purify SMRTbell Templates with 0.45X AMPure PB Beads, select size by using the BluePippin System, and repair DNA damage after size selection. Last, the library quality was assessed on the Qubit ${ }^{\circledR} 2.0$ Fluorometer (Thermo Fisher Scientific, Waltham, MA, USA), and the insert-fragment size was detected by Agilent 2100 (Agilent Technologies, Santa Clara, CA, USA). Sequencing libraries of Illumina NovaSeq platform were generated using NEBNext ${ }^{\circledR}$ Ultra $^{\text {TM }}$ DNA Library Prep Kit for Illumina (NEB, Ipswich, England), by following the manufacturer's recommendations; and index codes were added to attribute sequences to each sample. Briefly, the DNA sample was fragmented by sonication to a size of $350 \mathrm{bp}$, and then DNA fragments were end-polished, A-tailed, and ligated with the full-length adaptor for 
Illumina sequencing with further PCR amplification. Last, PCR products were purified using AMPure XP system (Beckman Coulter, Brea, CA, USA), and libraries were analyzed for size distribution by Agilent2100 (Agilent Technologies) and quantified using realtime PCR. The generated reads were assembled using SOAPdenovo (release 1.04). Genes were predicted by using Glimmer 3.02, and gene orthologs were determined by using OrthoMCL V1.4. The complete genome of strain BS26 (1,932,292 bp) contained 2022 genes and had a G+C content of $39.75 \%$. The draft genome of strain BS27 (1,909,795 bp) contained 2002 genes and had a G+C content of $39.69 \%$. The coverage/depth of BS26 and BS27 genomes were $100 \% / 288$ and $98.84 \% / 301$, respectively. For comparison, 11 draft genomes of $S$. parasuis strains were also added, including serotype 20 reference strain 86-5192, serotype 22 reference strain 88-1861, serotype 26 reference strain 89-4109-1, SUT-7, SUT-286, SUT-319, SUT-328, SUT-380, 2674, 4253 [35], and 10-36905 (Table 1).

\subsection{Phylogenetic Analysis of $16 S$ RNA and Housekeeping Genes}

Full-length $16 \mathrm{~S}$ rRNA, housekeeping genes groEL, gyrB, sodA, and $r e c N$ sequences of the S. parasuis were retrieved from the GenBank database or assembled genomes and aligned by the program CLUSTAL W. With the MEGA 7 software (available online: www. megasoftware.net, accessed on 27 February 2021), the 16 S rRNA and housekeeping genes' sequence-based phylogenetic trees were constructed with neighbor-joining algorithms. The stability of the groupings was estimated by bootstrap analysis at the level of 1000 replications, and the genetic distances were calculated by the Kimura 2-parameter method. The corresponding sequences of S. suis type strain NCTC10234 ${ }^{\mathrm{T}}$ (Accession no. PRJEB6403) were included. The corresponding sequences of Enterococcus faecalis JCM 5803 (Accession no. NR_040789.1) or S. suis type strain NCTC10234 ${ }^{\mathrm{T}}$ were used as out-group to root the trees.

\subsection{Experimental Infection}

\subsubsection{Survival Assay}

For comparison, a highly pathogenic S. suis strain P1/7 that was isolated from a pig with meningitis [11] was included in the present study. C57BL/ 6 mice (6 weeks old, female) were injected intraperitoneally with $5 \times 10^{7} \mathrm{CFU}$ of the live S. parasuis strain BS26, S. parasuis strain BS27, and S. suis strain P1/7 in $1 \mathrm{~mL}$ PBS or $1 \mathrm{~mL}$ PBS only as control group. Each strain-infected group contained ten mice and the mock-infected group contained five mice. The mortality was recorded per two hours within $12 \mathrm{~h}$ post-infection and per six hours from $12 \mathrm{~h}$ to $72 \mathrm{~h}$ post-infection. The experiment was performed independently in duplicate. Overall survival rates of the two experiments for each infected group were calculated via the Kaplan-Meier method.

\subsubsection{Histopathological Analysis and Bacterial Loads in Survival Mice}

In survival assay, the peripheral blood, brain, lung, liver, spleen, and kidney of all survival mice at $72 \mathrm{~h}$ post-infection (three mice infected with S. parasuis strain BS26, five mice infected with S. parasuis strain BS27 and three mice infected with S. suis strain P1/7) were collected aseptically.

One hundred microliters of serial tenfold dilutions of peripheral blood of each infected mouse were plated onto blood agar plates. Part of organs were accurately weighted and thoroughly ground to homogenate, placed in $1 \mathrm{~mL}$ of PBS. One hundred microliters of serial tenfold dilutions of organ homogenate were plated onto blood agar plates. Colonies were expressed as CFU/0.1 g for organ samples or CFU/mL for blood samples. The median values were used to express the bacterial counts of each group.

Another part of organs was fixed for $24 \mathrm{~h}$ at room temperature in $4 \%$ buffered formalin. After paraffin embedding, tissue sections conventional dewaxing to water were stained with H\&E and dehydrated according to standard protocol. The images were acquired and analyzed under light microscopy. 


\subsubsection{In Vivo Cytokine Production and Measurement of Bacterial Loads}

C57BL/ 6 mice (6 weeks old, female) were injected intraperitoneally with $1 \times 10^{7} \mathrm{CFU}$ of the live S. parasuis strain BS26 and S. suis strain P1/7 strains in $1 \mathrm{~mL}$ PBS. Each group contained five mice. At $4 \mathrm{~h}, 8 \mathrm{~h}, 12 \mathrm{~h}, 16 \mathrm{~h}$, and $24 \mathrm{~h}$ post-infection, the mice were killed, and the peripheral blood of each infected mouse was collected aseptically. These experiments were performed twice. All serum samples were tested with concentrations of IL-6 and TNF- $\alpha$ using the ELISA kit (R\&D Systems, Minneapolis, MN, USA), as recommended by the manufacturer. The cytokine values were expressed as the median $\mathrm{pg} / \mathrm{mL}$ values. The bacterial counts in peripheral blood, liver, lung, and brain of infected mice were measured according to the methods described in 4.4.2. The median values were used to express the bacterial counts of each group.

\subsection{Detection of Antibiotic Resistance (AR) Determinants and Antimicrobial Susceptibility Profiles}

We analyzed AR genes by searching the Comprehensive Antibiotic Resistance database (CARD) and the Antibiotic Resistance genes database (ARDB). We regarded a resistance gene as a homolog in the tested strains only if it exhibited at least $80 \%$ identity in the protein's sequence across $80 \%$ of the protein's length [36]. We tested for antimicrobial susceptibility by assessing the minimum inhibitory concentration (MIC) for all isolates by using an MIC-test strip (Liofilchem, Roseto degli Abruzzi, Italy). Those strips had a range of concentrations of penicillin $\mathrm{G}(0.002-32 \mu \mathrm{g} / \mathrm{mL})$, cefaclor $(0.016-256 \mu \mathrm{g} / \mathrm{mL})$, tetracycline $(0.016-256 \mu \mathrm{g} / \mathrm{mL})$, erythromycin $(0.016-256 \mu \mathrm{g} / \mathrm{mL})$, azithromycin $(0.016-256 \mu \mathrm{g} / \mathrm{mL})$, clindamycin $(0.016-256 \mu \mathrm{g} / \mathrm{mL})$, chloramphenicol $(0.016-256 \mu \mathrm{g} / \mathrm{mL})$, vancomycin $(0.016-$ $256 \mu \mathrm{g} / \mathrm{mL})$, streptomycin $(0.064-1024 \mu \mathrm{g} / \mathrm{mL})$, kanamycin $(0.016-256 \mu \mathrm{g} / \mathrm{mL})$, spectinomycin $(0.064-1024 \mu \mathrm{g} / \mathrm{mL})$, gentamicin $(0.016-256 \mu \mathrm{g} / \mathrm{mL})$, and trimethoprim-sulfamethoxazole $(0.002-32 \mu \mathrm{g} / \mathrm{mL})$. For quality control, we used S. pneumoniae ATCC 49619. For penicillin $G$, tetracycline, azithromycin, erythromycin, clindamycin, vancomycin, and chloramphenicol, breakpoints were used as recommended by the Clinical and Laboratory Standard Institute (CLSI) guidelines 2019 (M100-S29) for Streptococcus spp. Viridans group. For cefaclor and trimethoprim-sulfamethoxazole, breakpoints were used as recommended by the Clinical and Laboratory Standard Institute (CLSI) guidelines 2019 (M100-S29) for S. pneumoniae. No breakpoint values of streptomycin, kanamycin, gentamicin, and spectinomycin were available for Streptococcus. Their breakpoints were taken from previous studies of S. suis [37,38]. Breakpoints of antimicrobial agents are as follows: penicillin $\mathrm{G},>4 \mu \mathrm{g} / \mathrm{mL}$; cefaclor, $>4 \mu \mathrm{g} / \mathrm{mL}$; tetracycline, $>8 \mu \mathrm{g} / \mathrm{mL}$; azithromycin, $>2 \mu \mathrm{g} / \mathrm{mL}$; erythromycin, $>1 \mu \mathrm{g} / \mathrm{mL}$; clindamycin, $>1 \mu \mathrm{g} / \mathrm{mL}$; chloramphenicol, $>16 \mu \mathrm{g} / \mathrm{mL}$; vancomycin, $>2 \mu \mathrm{g} / \mathrm{mL}$; trimethoprim-sulfamethoxazole, $>4 \mu \mathrm{g} / \mathrm{mL}$; streptomycin, $>250 \mu \mathrm{g} / \mathrm{mL}$; kanamycin, $>250 \mu \mathrm{g} / \mathrm{mL}$; gentamicin, $>250 \mu \mathrm{g} / \mathrm{mL}$, and spectinomycin, $>250 \mu \mathrm{g} / \mathrm{mL}$.

\subsection{Comparative Genomic Analysis of S. parasuis}

Thirteen S. parasuis genomes and genome of S. suis type strain $\mathrm{NCTC} 10234^{\mathrm{T}}$ were included in the comparative genomic analysis. The average nucleotide identity (ANI) analysis was performed on the OrthoANIu platform (available online: https:/ / www.ezbiocloud. net/tools/orthoaniu, accessed on 20 November 2020) to define species boundaries of bacteria [39].

The whole genome phylogenetic tree based on 442 core genes of 14 genomes was constructed by Roary [40]. The core genes were extracted by BLASTP ( $96 \%$ identity, $99 \%$ of isolates a gene must be in to be core). The sequences of core genome were concatenated and aligned by MAFFT multiple sequence alignment program. The phylogenetic tree based on core genome was constructed by using the maximum likelihood algorithms in FastTree. The S. suis type strain NCTC10234 ${ }^{\mathrm{T}}$ was used as out-group to root the tree. 


\subsection{Analysis of cps Loci}

Each $c p$ s locus sequence was extracted from the genomes. The TMHMM v2.0 analysis program (available online: http://www.cbs.dtu.dk/services/TMHMM/, accessed on 15 October 2020) was used to identify putative transmembrane proteins WZX and WZY. The wzy genes having a global match region at $<50 \%$ of the amino-acid sequence and with an identity of $<50 \%$ were identified as different $c p s$ types in pairwise comparisons between S. parasuis genomes. The sequence comparison was performed using blastN program in BLAST with an e-value cutoff of e-10 and was visualized using an in-house Perl script (available online: https:/ / github.com/dupengcheng/BlastViewer, accessed on 2 March 2021).

\subsection{Statistics}

The survival rates of different groups were compared using Log-rank test. Significant difference in bacterial counts between $S$. suis and S. parasuis-infected group was determined by Wilcoxon's two-sample test. Statistical analysis of the cytokine data was performed by using the Wilcoxon's two-sample test. For these tests, a $p$-value $<0.05$ was considered to be significant.

\subsection{Nucleotide Sequence Accession Numbers}

The sequences of the $2 \mathrm{~S}$. parasuis strains that were sequenced in the study were deposited in the GenBank under accession numbers CP069079 (BS26) and JAETXU000000000 (BS27).

Supplementary Materials: The following are available online at https:/ /www.mdpi.com/article/10 $.3390 /$ pathogens10070834/s1. Table S1. Sequence similarity of 16S rRNA gene and housekeeping genes and ANI values among genomes that the present study used. Table S2. Survival table of each infected group and statistical data in survival assay.

Author Contributions: Conceptualization, H.Z.; methodology, H.Z., J.W., P.L., and Y.W.; validation, D.J. and J.Y.; formal analysis, Y.T., Y.W., D.J., and J.Y.; investigation, X.Y. and B.L.; resources, X.Y. and B.L.; data curation, H.Z.; writing—original draft preparation, J.W. and P.L.; writing—review and editing, H.Z.; supervision, H.Z.; funding acquisition, H.Z. All authors have read and agreed to the published version of the manuscript.

Funding: This work was supported by the Priority Project on Infectious Disease Control and Prevention from the Ministry of Science and Technology of the People's Republic of China [Grant No. 2017ZX10303405-002, 2018ZX10734404] and the National Natural Science Foundation of China [Grant No. 81572044].

Institutional Review Board Statement: The study was approved by the ethics committee of the National Institute for Communicable Disease Control and Prevention, Chinese Center for Disease Control and Prevention (protocol code 2020-021 and 29-12-2020 of approval).

Informed Consent Statement: Informed consent was obtained from all patients to collect clinical strains and related information used in the study.

Data Availability Statement: The data presented in this study are openly available in the article and its Supplementary Material.

Conflicts of Interest: The authors declare no conflict of interest.

\section{References}

1. Goyette-Desjardins, G.; Auger, J.P.; Xu, J.; Segura, M.; Gottschalk, M. Streptococcus suis, an important pig pathogen and emerging zoonotic agent-an update on the worldwide distribution based on serotyping and sequence typing. Emerg. Microbes Infect. 2014, 3, e45. [CrossRef]

2. Gajdács, M.; Németh, A.; Knausz, M.; Barrak, I.; Stájer, A.; Mestyán, G.; Melegh, S.; Nyul, A.; Tóth, Á.; Ágoston, Z.; et al. Streptococcus suis: An Underestimated Emerging Pathogen in Hungary? Microorganisms 2020, 8, 1292. [CrossRef] [PubMed]

3. Ágoston, Z.; Terhes, G.; Hannauer, P.; Gajdács, M.; Urbán, E. Fatal case of bacteremia caused by Streptococcus suis in a splenectomized man and a review of the European literature. Acta Microbiol. Immunol. Hung. 2020, 67, 148-155. [CrossRef] 
4. Hill, J.E.; Gottschalk, M.; Brousseau, R.; Harel, J.; Hemmingsen, S.M.; Goh, S.H. Biochemical analysis, cpn60 and $16 \mathrm{~S}$ rDNA sequence data indicate that Streptococcus suis serotypes 32 and 34, isolated from pigs, are Streptococcus orisratti. Vet. Microbiol. 2005, 107, 63-69. [CrossRef]

5. Nomoto, R.; Maruyama, F.; Ishida, S.; Tohya, M.; Sekizaki, T.; Osawa, R. Reappraisal of the taxonomy of Streptococcus suis serotypes 20, 22 and 26: Streptococcus parasuis sp. nov. Int. J. Syst. Evol. Microbiol. 2015, 65, 438-443. [CrossRef] [PubMed]

6. Yamada, R.; Tien, L.H.T.; Arai, S.; Tohya, M.; Ishida-Kuroki, K.; Nomoto, R.; Kim, H.; Suzuki, E.; Osawa, R.; Watanabe, T.; et al. Development of PCR for identifying Streptococcus parasuis, a close relative of Streptococcus suis. J. Vet. Med. Sci. 2018, 80, 1101-1107. [CrossRef] [PubMed]

7. Gottschalk, M.; Lacouture, S. Canada: Distribution of Streptococcus suis (from 2012 to 2014) and Actinobacillus pleuropneumoniae (from 2011 to 2014) serotypes isolated from diseased pigs. Can. Vet. J. 2015, 56, 1093-1094.

8. Gottschalk, M.; Lacouture, S.; Bonifait, L.; Roy, D.; Fittipaldi, N.; Grenier, D. Characterization of Streptococcus suis isolates recovered between 2008 and 2011 from diseased pigs in Québec, Canada. Vet. Microbiol. 2013, 162, 819-825. [CrossRef]

9. Wei, Z.; Li, R.; Zhang, A.; He, H.; Hua, Y.; Xia, J.; Cai, X.; Chen, H.; Jin, M. Characterization of Streptococcus suis isolates from the diseased pigs in China between 2003 and 2007. Vet. Microbiol. 2009, 137, 196-201. [CrossRef]

10. Gottschalk, M.; Higgins, R.; Jacques, M.; Beaudoin, M.; Henrichsen, J. Characterization of six new capsular types (23 through 28) of Streptococcus suis. J. Clin. Microbiol. 1991, 29, 2590-2594. [CrossRef]

11. Holden, M.T.G.; Hauser, H.; Sanders, M.; Ngo, T.H.; Cherevach, I.; Cronin, A.; Goodhead, I.; Mungall, K.; Quail, M.A.; Price, C.; et al. Rapid Evolution of Virulence and Drug Resistance in the Emerging Zoonotic Pathogen Streptococcus suis. PLoS ONE 2009, 4, e6072. [CrossRef]

12. Okura, M.; Osaki, M.; Nomoto, R.; Arai, S.; Osawa, R.; Sekizaki, T.; Takamatsu, D. Current Taxonomical Situation of Streptococcus suis. Pathogens 2016, 5, 45. [CrossRef] [PubMed]

13. Niu, L.; Lu, S.; Hu, S.; Jin, D.; Lai, X.; Yang, J.; Chen, C.; Wang, Y.; Wang, Y.; Bai, X.; et al. Streptococcus marmotae sp. nov., isolated from the respiratory tract of Marmota himalayana. Int. J. Syst. Evol. Microbiol. 2016, 66, 4315-4322. [CrossRef] [PubMed]

14. Du, H.; Huang, W.; Xie, H.; Ye, C.; Jing, H.; Ren, Z.; Xu, J. The genetically modified suilysin, rSLYP353L, provides a candidate vaccine that suppresses proinflammatory response and reduces fatality following infection with Streptococcus suis. Vaccine 2013, 31, 4209-4215. [CrossRef]

15. Domínguez-Punaro, M.C.; Segura, M.; Plante, M.-M.; Lacouture, S.; Rivest, S.; Gottschalk, M. Streptococcus suis Serotype 2, an Important Swine and Human Pathogen, Induces Strong Systemic and Cerebral Inflammatory Responses in a Mouse Model of Infection. J. Immunol. 2007, 179, 1842-1854. [CrossRef] [PubMed]

16. Lachance, C.; Gottschalk, M.; Gerber, P.P.; Lemire, P.; Xu, J.; Segura, M. Exacerbated Type II Interferon Response Drives Hypervirulence and Toxic Shock by an Emergent Epidemic Strain of Streptococcus suis. Infect. Immun. 2013, 81, 1928-1939. [CrossRef] [PubMed]

17. Okura, M.; Takamatsu, D.; Maruyama, F.; Nozawa, T.; Nakagawa, I.; Osaki, M.; Sekizaki, T.; Gottschalk, M.; Kumagai, Y.; Hamada, S. Genetic Analysis of Capsular Polysaccharide Synthesis Gene Clusters from All Serotypes of Streptococcus suis: Potential Mechanisms for Generation of Capsular Variation. Appl. Environ. Microbiol. 2013, 79, 2796-2806. [CrossRef]

18. Yarza, P.; Richter, M.; Peplies, J.; Euzeby, J.; Amann, R.; Schleifer, K.-H.; Ludwig, W.; Glöckner, F.O.; Rosselló-Móra, R. The All-Species Living Tree project: A $16 \mathrm{~S}$ rRNA-based phylogenetic tree of all sequenced type strains. Syst. Appl. Microbiol. 2008, 31, 241-250. [CrossRef]

19. Zhang, W.; Du, P.; Zheng, H.; Yu, W.; Wan, L.; Chen, C. Whole-genome sequence comparison as a method for improving bacterial species definition. J. Gen. Appl. Microbiol. 2014, 60, 75-78. [CrossRef]

20. Goris, J.; Konstantinidis, K.T.; Klappenbach, J.A.; Coenye, T.; Vandamme, P.; Tiedje, J.M. DNA-DNA hybridization values and their relationship to whole-genome sequence similarities. Int. J. Syst. Evol. Microbiol. 2007, 57, 81-91. [CrossRef]

21. Ye, C.; Zheng, H.; Zhang, J.; Jing, H.; Wang, L.; Xiong, Y.; Wang, W.; Zhou, Z.; Sun, Q.; Luo, X.; et al. Clinical, Experimental, and Genomic Differences between Intermediately Pathogenic, Highly Pathogenic, and EpidemicStreptococcus suis. J. Infect. Dis. 2009, 199, 97-107. [CrossRef]

22. Dofferhoff, A.S.; Vellenga, E.; Limburg, P.C.; Van Zanten, A.; Mulder, P.O.; Weits, J. Tumour necrosis factor (cachectin) and other cytokines in septic shock: A review of the literature. Neth. J. Med. 1991, 39, 45-62.

23. Norrby-Teglund, A.; Pauksens, K.; Norgren, M.; Holm, S.E. Correlation between Serum TNF $\alpha$ and IL6 levels and Severity of Group: A Streptococcal Infections. Scand. J. Infect. Dis. 1995, 27, 125-130. [CrossRef] [PubMed]

24. Faulkner, L.; Cooper, A.; Fantino, C.; Altmann, D.; Sriskandan, S. The Mechanism of Superantigen-Mediated Toxic Shock: Not a Simple Th1 Cytokine Storm. J. Immunol. 2005, 175, 6870-6877. [CrossRef]

25. Zheng, H.; Ye, C.; Segura, M.; Gottschalk, M.; Xu, J. Mitogenic effect contributes to increased virulence of Streptococcus suis sequence type 7 to cause streptococcal toxic shock-like syndrome. Clin. Exp. Immunol. 2008, 153, 385-391. [CrossRef] [PubMed]

26. Wang, M.; Du, P.; Wang, J.; Lan, R.; Huang, J.; Luo, M.; Jiang, Y.; Zeng, J.; Quan, Y.; Shi, Z.; et al. Genomic Epidemiology of Streptococcus suis Sequence Type 7 Sporadic Infections in the Guangxi Zhuang Autonomous Region of China. Pathogens 2019, 8, 187. [CrossRef]

27. Palmieri, C.P.; Varaldo, P.E.M.; Facinelli, B.P. Streptococcus suis, an Emerging Drug-Resistant Animal and Human Pathogen. Front. Microbiol. 2011, 2, 235. [CrossRef] [PubMed] 
28. Huang, J.; Ma, J.; Shang, K.; Hu, X.; Liang, Y.; Li, D.; Wu, Z.; Dai, L.; Chen, L.; Wang, L. Evolution and Diversity of the Antimicrobial Resistance Associated Mobilome in Streptococcus suis: A Probable Mobile Genetic Elements Reservoir for Other Streptococci. Front. Cell. Infect. Microbiol. 2016, 6, 118. [CrossRef]

29. Yun, K.W.; Cho, E.Y.; Choi, E.H.; Lee, H.J. Capsular polysaccharide gene diversity of pneumococcal serotypes 6A, 6B, 6C, and 6D. Int. J. Med Microbiol. 2014, 304, 1109-1117. [CrossRef]

30. Liu, Z.; Zheng, H.; Gottschalk, M.; Bai, X.; Lan, R.; Ji, S.; Liu, H.; Xu, J. Development of Multiplex PCR Assays for the Identification of the 33 Serotypes of Streptococcus suis. PLoS ONE 2013, 8, e72070. [CrossRef]

31. Baker, G.; Smith, J.; Cowan, D. Review and re-analysis of domain-specific 16S primers. J. Microbiol. Methods 2003, 55, 541-555. [CrossRef]

32. Okwumabua, O.; O'Connor, M.; Shull, E. A polymerase chain reaction (PCR) assay specific forStreptococcus suisbased on the gene encoding the glutamate dehydrogenase. FEMS Microbiol. Lett. 2003, 218, 79-84. [CrossRef]

33. Tien, L.H.T.; Nishibori, T.; Nishitani, Y.; Nomoto, R.; Osawa, R. Reappraisal of the taxonomy of Streptococcus suis serotypes 20, 22, 26, and 33 based on DNA-DNA homology and sodA and recN phylogenies. Vet. Microbiol. 2013, 162, 842-849. [CrossRef] [PubMed]

34. Ishida, S.; Tien, L.H.T.; Osawa, R.; Tohya, M.; Nomoto, R.; Kawamura, Y.; Takahashi, T.; Kikuchi, N.; Kikuchi, K.; Sekizaki, T. Development of an appropriate PCR system for the reclassification of Streptococcus suis. J. Microbiol. Methods 2014, 107, 66-70. [CrossRef] [PubMed]

35. Stevens, M.J.A.; Cernela, N.; Corti, S.; Stephan, R. Draft Genome Sequence of Streptococcus parasuis 4253, the First Available for the Species. Microbiol. Resour. Announc. 2019, 8, e00203-19. [CrossRef]

36. Hu, Y.; Yang, X.; Qin, J.; Lu, N.; Cheng, G.; Wu, N.; Pan, Y.; Li, J.; Zhu, L.; Wang, X.; et al. Metagenome-wide analysis of antibiotic resistance genes in a large cohort of human gut microbiota. Nat. Commun. 2013, 4, 2151. [CrossRef] [PubMed]

37. Huang, K.; Zhang, Q.; Song, Y.; Zhang, Z.; Zhang, A.; Xiao, J.; Jin, M. Characterization of Spectinomycin Resistance in Streptococcus suis Leads to Two Novel Insights into Drug Resistance Formation and Dissemination Mechanism. Antimicrob. Agents Chemother. 2016, 60, 6390-6392. [CrossRef]

38. Marie, J.; Morvan, H.; Berthelot-Hérault, F.; Sanders, P.; Kempf, I.; Gautier-Bouchardon, A.V.; Jouy, E.; Kobisch, M. Antimicrobial susceptibility of Streptococcus suis isolated from swine in France and from humans in different countries between 1996 and 2000. J. Antimicrob. Chemother. 2002, 50, 201-209. [CrossRef] [PubMed]

39. Yoon, S.-H.; Ha, S.-M.; Lim, J.; Kwon, S.; Chun, J. A large-scale evaluation of algorithms to calculate average nucleotide identity. Antonie van Leeuwenhoek 2017, 110, 1281-1286. [CrossRef] [PubMed]

40. Page, A.J.; Cummins, C.A.; Hunt, M.; Wong, V.K.; Reuter, S.; Holden, M.T.G.; Fookes, M.; Falush, D.; Keane, J.A.; Parkhill, J. Roary: Rapid large-scale prokaryote pan genome analysis. Bioinformatics 2015, 31, 3691-3693. [CrossRef] 